\title{
Dealer's Ordering Decision Behavior under Different Price Strategies: An Analysis Based on the Modern Behavioral Decision Theory
}

\author{
Bingqun Cui ${ }^{1}$, Tingjui $\mathrm{Chou}^{2}$ \\ ${ }^{1}$ School of Economics and Management, Southwest Jiaotong University, Chengdu, China \\ ${ }^{2}$ Cirrus Data Insights Ltd., Taiwan, China \\ Email: bingquncui@163.com
}

Received 8 April 2016; accepted 23 April 2016; published 27 April 2016

Copyright (C) 2016 by authors and Scientific Research Publishing Inc.

This work is licensed under the Creative Commons Attribution International License (CC BY).

http://creativecommons.org/licenses/by/4.0/

(c) (i) Open Access

\begin{abstract}
The modern behavioral decision theory considers that the decision subject is often in a state of bounded rationality rather than absolute rationality. From the perspective of decision behavior, this thesis based on the prospect theory deeply parses dealer's ordering decision behavior in response to different price strategies under the random market demand, reveals the intrinsic mechanism of dealer's ordering decision behavior in response to business price strategy, analyzes the non-pricing factors impacting dealer's ordering decision under the price strategy, and proposes the countermoves and suggestions for enterprise in terms of business price strategy, product strategy, relationship marketing and other aspects, which will be of some references to guide the application of business price strategy and channel management practices.
\end{abstract}

\section{Keywords}

Price Strategy, Ordering Decision Behavior, Modern Behavioral Decision Theory

\section{Introduction}

In business marketing practices, more and more enterprises implement various price strategies for their dealers. In the context of random market demand, dealer may make different ordering decision behaviors in response to different price strategies, based on the available information, customer needs, experience-based judgment and others. With the scientific development of modern decision behavior, the behavioral decision theory has been extensively applied in economic, managerial and marketing sectors, and relevant studies on dealer's ordering decision behavior has aroused the growing attention of marketing scholars. It is worthy of further discussing 
whether dealer decides to order or not, dealer's frequent ordering decision, and to which extent the ordering quantity decision will be subject to price strategies.

From the scientific perspective of modern behavioral decision, this thesis based on the expected utility and prospect theory deeply parses the intrinsic mechanism of dealer's ordering decision behavior in response to business price strategy, makes clear various factors impacting dealer's ordering decision under the price strategy, further discusses how an enterprise deals with and utilizes these factors to develop the scientific combination of strategies in business marketing management, and offers the theoretical basis and practical guidance for the promotion of channel sales.

\section{Literature Review}

With the continuous development of society, there is a growing public attention on behavioral decision theory. According to the scientific development course of behavioral decision, relevant studies of the behavioral decision theory witness the transition from the earliest rational assumption-based maximum expected utility theory, the subjective expected utility theory, to the prospect theory under the bounded rational assumption [1]. The early relevant studies of behavioral decision were conducted through the expected utility theory proposed by Neumann and Morgenstern (1944) who believed that the subject of behavioral decision is absolutely rational with same hobbies and interests as well as complete information, namely every decision-maker is able to gain the objective information about his product demand from the market at any time, also referred to as the hypothesis of rational man. With such hypothesis, the "judgment" and "choice" by the decision subject follow the maximum expected utility principle. However, this is obviously an ideal state. The decision behavior in an extremely simple decision-making environment seems to be consistent with the maximum expected utility theory, but some deviation may appear between the actual decision behavior and the maximum expected utility principle when the decision-making environment becomes a little more complex. The famous American managerialist and sociologist Simon's view has a huge influence on the sciences of modern behavioral decision, who claimed that information uncertainty, complexity and unknown risks will restrain the rational decision by the decision subject. Therefore, the decision subject is often in a state of bounded rationality rather than absolute rationality [2].

As the most representative theory in the study area of modern descriptive decision behavior, the prospect theory proposed by Kahneman and Tversky (1979) [3] has been extensively applied over these years, and its principles mainly comprise "framing effects", "reference point dependence”, “decision weight” and "value function". This theory argues that the decision subject will set a reference point in the process of behavioral decision, determines "gain" or "loss" according to the levels higher or lower than the reference point correspondingly, collect and process the information according to the reference point, framework and others, and judge the information through the decision weight and value function of its subjective probability. The price function also indicates that people's decision behavior usually shows three characteristics as risk aversion, reference point effect and diminishing marginal utility under the condition of risk uncertainty. Afterwards, the development of prospect theory also asserts that the delivery of value aims to pass on "gain" and "loss" instead of final asset, the value of each result isn’t affected by probability but the “decision weight” conferred by people's decision-making, also referred to as "cumulative prospect theory", a study area that makes the prospect theory better adapt to the dynamic changes.

\section{Analysis of Dealer's Ordering Decision Behavior}

As one of the important channel members that connect manufacturer and consumer, dealer's ordering decision has a significant impact on its profitability, customer service and enterprise production marketing management. Previous studies of dealer's ordering decision examine the effects of dealer's ordering decision on its profitability or establish the ordering model based on the decision theory. While Tony Haitao Cui (2008) [4] analyzed dealer may adopt different ordering decision to gain profits in a response to the price strategy based on the levels of its stockholding cost, from a different angle that the size of its inventory capacity results in the stockholding cost. By the research paradigm given above, dealer's ordering decision behaviors can be classified into whether dealer decides to order or not, dealer's frequent ordering decision, and change decision for single ordering quantity in a response to different pricing strategies, from the perspective of behavioral decision, according to the 
change of dealer's total ordering quantity affected by the ordering decision.

\subsection{Whether Dealer Decides to Order or Not}

According to the principle of "reference point dependence" [3], the starting point whether dealer decides to order or not is not only related to the absolute value of price, generally speaking, the decision-maker also sets a reference price for its product, also known as the reference point, and compares the future decision result with the reference price to determine gain or loss. With one price strategy, the transaction will be perceived to be more cost-effective if he perceives the ordering price of the designated product is lower than the reference price, he is more likely to make the ordering decision. According to the prospect theory principle that manufacturer's price strategy expression approach causes "framing effects" on dealer, if information with the same content is expressed in different ways, it will produce different perception impact on people. For example, the price reduction strategy will make customer perceived of gaining "profits" from the ordering of the designated product, while the price rise strategy will make customer perceived of suffering "losses" from the ordering. Thus, when a dealer receives the enterprise price strategy, he may tend to make the ordering decision in the price reduction strategy, compared to the price rising strategy. "Framing effects" have been extensively used in enterprise pricing or promotion and other price strategies. Nonetheless, due to the presence of marginal personnel behaviors between enterprise and dealer, for example, the sales manager in contact with dealer on behalf of enterprise, many empirical studies have indicated that whether dealer decides to order or not is often subject to the personnel service levels, expression approach of strategy, relationship strategy, and his relationship with other dealers by sales manager and others in direct contact with him. This also supports that dealer's ordering decision behavior belongs to irrational decision behaviors, which is a very typical individual decision behavior.

\subsection{Dealer's Frequent Ordering Decision}

"Value function" in prospect theory can be described as one "S" curve including "gains" and "losses", the value function of "gains" is in concave shape while the value function of "losses" is in convex shape, the reference point is the inflection point of curve, but the response of decision-maker to the expected "losses" is more sensitive than that to the expected gains, which means that the response of customer to the price higher than the reference point is more intense than that to the price lower than the reference point. Subject to the impact of reference point, dealer may have different psychological experiences in case of "gains" or "losses", and tends to choose the definitive result for gains; but take a risk for losses. However, once a dealer who extends cooperation with enterprise decides to place an order, he is likely to choose the frequent ordering behavior to increase the total ordering quantity in response to the price strategy of expected "losses", due to the defined inventory capacity or the stockholding cost is relatively fixed. From another point of view, since the dealer often maintains the long-term and repetitive cooperation with enterprise, he cannot determine whether the expected "losses" can be also passed to consumers by means of "price rising" in a response to one price rising strategy of expected "losses", who may gradually implement the information publicity and behavioral performance of "price rising" in the following sales process, and in this way dealer will not increase the ordering quantity while satisfying the normal consumer demand, but merely makes frequent ordering decision behaviors to deal with it. According to "the cumulative prospect theory", dealer may make the frequent ordering decisions in various degrees, subject to the impact of "decision weight" in every decision-making process [5].

\subsection{Change Decision for Single Ordering Quantity}

According to the viewpoints of White (2002) [6] and Yeshin (2006) [7], combined with the business channel price practice, it is considered that the main objectives of enterprise implementing price strategies for dealer include: promoting the selling of new products; feedback existing customers to resist against the competition; stimulate the repetitive and frequent ordering of products; encourage dealer to place large orders; and ensure dealer maintain the sufficient inventory and product display. As for dealer, he gains all profits by selling its products to end consumers regardless of the ordering quantity, which means that total ordering levels are subject to the impact of his sales or the inventory turnover rate. Thus, total ordering levels of dealer within a certain period depend on the ordering frequency and single ordering quantity.

With respect to the "framing effects" principle, a simple example is illustrated here: in enterprise price strategy, the pricing for Brand S product is RMB $20 / \mathrm{m}^{2}$, but dealer will be notified "if the ordering quantity of this 
product within the designated period exceeds the specified quantity, the unit price per $\mathrm{m}^{2}$ will be reduced by RMB 5"; for Brand W product, the pricing is RMB $15 / \mathrm{m}^{2}$, but dealer will be notified "if the ordering quantity of this product within the designated period is smaller than the specified quantity, the unit price per $\mathrm{m}^{2}$ will be increased by RMB 5”. In fact, the economic costs borne by dealer for Brand S and Brand W are identical. However, the effects and impacts of these two price strategies may vary from each other, the price expression for Brand $\mathrm{S}$ will make dealer perceived of "gains" if the ordering quantity increases, while the price expression for Brand W will make dealer perceived of more "losses” if the ordering quantity doesn't increase.

This may lead to different decision behaviors of dealer to whether increase the single ordering quantity or not, generally speaking, it is more likely for dealer to make the decision behavior to increase the single ordering quantity for Brand S than Brand W. As the price promotion by manufacturer generally targets at all or most dealers, there is a competition among dealers during the selling process of products, and with the implementation of manufacturer's price strategy, as the time extends, dealer's sales price may be reduced to some extent after he gains the price discount conferred by manufacturer, so as to increase the sales quantity and gain higher profits. For this reason, dealer will get higher price difference to gain more profits if he could purchase goods from manufacturer and conclude the selling as earlier as possible and as much as possible, which will result in the increase of single ordering quantity. Therefore, dealer's single ordering levels will increase in a response to one type of price strategies, generally speaking, he will make a decision to increase the purchase quantity to ensure the sufficient inventory immediately after the receipt of price reduction, so that he could gain profits as early as possible after the selling of promotion products, which will lead to dealer's decision behavior for the increase of single ordering quantity.

\section{Analysis of Non-Price Factors of Dealer's Ordering Decision Behavior}

As noted in the above analysis, when a dealer faces with the business price strategies, the direct price factors such as mode of price expression, intensity of price adjustment, reference price and relative price play a key role in impacting his ordering decision. Nonetheless, as the decision behavior is bounded rationality even irrational, the factors impacting dealer's ordering decision behavior tend to be more complex and diverse, from the scientific studies of modern behavioral decision. Combined with business marketing practices, the non-price factors impacting dealer's ordering decision behavior under the price strategies are summarized below.

Firstly, although price plays an important role in dealer's decision, some non-price factors associated with price also plays a crucial role. For example, enterprise presence and brand influence in the industry, the objective motive of implementing price strategies, the timing and time of implementing strategies, the information transmission and representation of price strategies and other factors will have a direct bearing with dealer's ordering decision behavior. For instance, enterprise adopts the price strategy of clearing the stocks of one product at an ultra-low price before the imminent peak season, in order to rapidly reduce the inventory within a certain period. Due to the limited inventory of this product and the imminent peak season, and such information is only passed to some loyal distributors with large inventory capacity and in long-term cooperation through the internal channels, dealer usually makes a quick decision to place order, even increase the single ordering quantity in such a case.

Secondly, some factors associated with dealer's own characteristics, such as dealer's operating scale and strength, operating characteristics, the individual demographic variables of dealer's decision-maker including sex, age and educational background will have a significant impact on dealer's ordering decision behavior. Empirical studies show that dealer's perceived justice may impact the channel relationship quality, while the channel relationship quality may also directly impact dealer's ordering decision behavior. In price strategies, different prices have a direct bearing with dealer’s actual expenditure, specifically the "acquisition utility”, but with the same price, different modes of promotion expression or implementation ways of strategies may impact "transaction utility" of customer, namely customer's perceived utility in the transaction process. The different perceived utilities will also have a direct bearing on dealer's ordering decision. Therefore, dealer's ordering decision behavior is also subject to the perception and other psychological factors and the extent of dealer's preference and loyalty for one business product, and dealer's ordering motive, attitude and other factors.

Additionally, according to the modern relationship marketing theory, especially in Chinese cultural context, the effects of relationship on commercial activity is a commonplace. Recent studies have reported or proved that the relationship status, relationship quality between enterprise and dealer, and the cross-organizational private 
relationship between marginal personnel who is in direct contact with dealers on behalf of enterprise and dealers, even the individual characteristics of enterprise marginal personnel and other factors will have a significant impact on the channel cooperation relationship and dealer's ordering decision behavior. With a view to the same information of price strategy, when the sales personnel who maintains a close relationship with dealer passes it to dealer and adopts the appropriate strategy and expression mode for the purpose of tracking and service, if he urges the dealer to place an order, the dealer may easily make the decision to order, frequent ordering decision or increase the single ordering quantity.

Therefore, as noted in the above analysis, it is not the single price factors that impact dealer's ordering decision, even under different price strategies. This gives an important hint to business managers in the implementation of price strategies, channel relationship management and other aspects. From the scientific perspective of behavioral decision, dealer's performance of the ordering decision is out of bounded rationality and subject to a multitude of complex factors.

\section{Business Marketing Countermoves and Suggestions}

According to the above analysis of dealer's ordering decision behavior under price strategies, in consideration of the hypothesis of rational man of the decision-making subject, enterprise managers should be inspired to not only emphasize the implementation of price strategies but also seek marketing strategies in dealer's price decision process based on the hypothesis of rational man for the benefit of business marketing channel management. Accordingly, the countermoves and suggestions for dealer's ordering decision behavior are proposed for enterprise under price strategies with respect to price strategies, product strategies, relationship marketing strategies and other aspects.

1) Flexibly apply the price strategies and effectively play its key role in impacting dealer's ordering decision.

Although the price strategy has been proved to be a very effective channel incentive policy and is extensively applied in business marketing practice, it is nothing but the "last resort" to some extent for enterprise. With the frequent application of price strategies, dealer may become somewhat "strategy dependent" that is worthy of being vigilant. As a matter of fact, enterprise may implement effective price discrimination for its dealers by launching various price strategies specifically for different dealers to achieve the aim of promoting sales and delivering profits to dealers. Combined with inference, several suggestions are further proposed below for the business application of price strategies: First, enterprise should define the goal for each price strategy and take the appropriate approach according to its realities, in consideration of production, inventory, goods supply and other conditions, according to the "framing effects" of price strategies, enterprise should examine different expression modes of price strategies and information transmission, and exercise the effective incentive effects of price strategies on dealer's ordering decision. Second, enterprise should consider all realities of different dealers to determine the price strategy. For example, dealers based in different regions may vary in selling pace, inventory capacity, stockholding cost and ordering habit, subject to various factors including the levels of economic development and product demand. In such a case, it is not advised to implement a price strategy for all regions and dealers. Third, enterprise should effective seize the timing and frequency of implementing the price strategy, in case of frequent implementation, on the one hand, it may easily lead to dealer's "strategic dependence", which is to the disadvantage of product orientation and brand value, and on the other hand, it will waste the business profits and cannot realize the promotional purpose.

2) Deeply research the product strategies and deal with the bounded rationality of dealer's ordering decision.

As dealer usually maintains a long-term cooperation with enterprise, most of his ordering behaviors are repeated and regular. Enterprise strengths, advantages and products are fundamental to dealer. Thus, enterprise should deeply research the product strategies, thoroughly provides such information as product quality, brand, selling point even the technical levels to dealer, shift the focus of dealer's ordering decision to the high-quality products and brands with big influence, and make dealer become more and more "rational" for the quality trust and brand recognition of business products. More importantly, dealers act as the "salesmen" and information transmitters of business products and more as the influencers of consumer's purchase decision, rather than the end consumers, the fundamental purpose of dealer's purchase is to make a profit. Therefore, the deep-going research of product strategies and the provision of marketable products that flexibly adapt to the market for dealers are fundamental to dealing with the bounded rationality of dealer's ordering decision. In addition, enterprise can 
effectively realize the rational use of "framing effects" combined with product category, size and brand and through the marketing elements such as packaging, advertising and decoration.

3) Fully implement the relationship marketing strategies and increase dealer's loyalty to enterprise.

With the deepening of relationship marketing studies, it has indicated that Chinese culture is different from western culture. In terms of commercial relationship, western countries emphasizes on the reciprocity principle, while China emphasizes one's position in the whole relationship network and different attitudes and behaviors for various positions. As one of the key components in social activities, enterprise marketing channel management is rooted in Chinese culture, which has Chinese characteristics. The relationship between enterprise and dealers or the cross-organizational private relationship between enterprise marginal personnel and dealers has a significant impact on bilateral cooperation. As one of the key components in channel relationship management, dealers play an important role in the whole channel ecosystem, and the bilateral relationship is essential and enterprise should pay attention to it. Once enterprise establishes a close and firm cooperation with dealers, their loyalty to enterprise will gradually increase, which in turn diverts dealer's ordering decision to the direction in favor of the enterprise and bilateral cooperation. Enterprise should communicate with dealer as much as possible, treat them as "one of its own members", get some suggestions for reference, and achieve better effect in implementing promotion strategies on grounds of mutual benefit and win-win result, while implementing price strategies.

The specific business price strategy for dealers can be also effectively combined with the specific price strategy for consumers to make an overall planning, make collocation with one another, serve the purpose of promoting dealer's purchase and selling, and only in this way could enterprise yield twice the result with half the effort and achieve the purpose of expanding the sales volume and increasing dealer's profits. In addition, the comprehensive tracking service covering dealer's product purchase, promotional cooperation and after-sales service and the fast satisfactory processing of dealer's demand serve as the keys to increase dealer's loyalty and effectively implementing the relationship marketing strategy.

\section{Conclusion}

In conclusion, the dependence between enterprise and dealers is increasingly inclined to dealers as the market competition is intensified. Enterprise has to take various incentive measures to get more orders from dealers and increase their loyalty levels. Through this study, based on relevant studies of dealer's ordering decision behavior under price strategies, the suggestions for business marketing strategies are proposed, which is of significance and practical value for manufacturers to effectively govern the channel relationship and expand their market shares.

\section{References}

[1] Wu, G., Zhou, J. and Lei, L. (2013) An Overview of Behavioral Decision Theory. Journal of Nanjing Tech University, 3, 101-105.

[2] Liu, J.Y. and Zhang, S.M. (2015) Review on Behavioral Decision-Making Theory with Uncertainty. Systems Engineering, 2, 110-117.

[3] Kahneman, D. and Tverskey, A. (1979) Prospect Theory: An Analysis of Decision under Risk. Econometrica, 47, 263-292. http://dx.doi.org/10.2307/1914185

[4] Cui, T.H., Raju, J.S. and Zhang, Z.J. (2008) A Price Discrimination Model of Trade Promotions. Marketing Science, 27, 779-795. http://dx.doi.org/10.1287/mksc.1070.0314

[5] Cui, B.Q. and Li, Y.P. (2015) Research on Mechanism of Distributors' Frequent Ordering Intention: A Prospect Theory Perspective. Modern Economy, 6, 1167-1172. http://dx.doi.org/10.4236/me.2015.611110

[6] White, R. (2002) Best Practice: Sales Promotions and the Brand. Admap, 436.

[7] Yeshin, T. (2006) Sales Promotion. Thomson Learning, London. 